\title{
Nucleosynthesis in dynamical and torus ejecta of compact binary mergers
}

\section{Oliver Just ${ }^{* 1,2}$}

E-mail: ojust@mpa-garching.mpg.de

\author{
A. Bauswein, ${ }^{3}$ R. Ardevol Pulpillo, ${ }^{1,4}$ S. Goriely ${ }^{5}$ and H.-T. Janka ${ }^{1}$ \\ ${ }^{1}$ Max-Planck-Institut für Astrophysik, Postfach 1317, 85741 Garching, Germany \\ ${ }^{2}$ Max-Planck/Princeton Center for Plasma Physics (MPPC) \\ ${ }^{3}$ Department of Physics, Aristotle University of Thessaloniki, 54124 Thessaloniki, Greece \\ ${ }^{4}$ Physik Department, Technische Universität München, James-Franck-Straße 1, 85748 Garching, \\ Germany \\ ${ }^{5}$ Institut d'Astronomie et d'Astrophysique, CP-226, Université Libre de Bruxelles, 1050 Brussels, \\ Belgium
}

\begin{abstract}
We present a comprehensive study of r-process element nucleosynthesis in the ejecta of compact binary mergers (CBMs) and their relic black-hole (BH)-torus systems. The evolution of the $\mathrm{BH}$-accretion tori is simulated for seconds with a Newtonian hydrodynamics code including viscosity, pseudo-Newtonian gravity for rotating BHs, and an energy-dependent two-moment closure neutrino transport scheme. The investigated cases are guided by relativistic double neutron star (NS-NS) and NS-BH merger models. Our nucleosynthesis analysis includes the dynamical ejecta expelled during the CBM phase and the neutrino and viscously driven outflows of the relic BH-torus systems. While typically $\sim 20-25 \%$ of the initial torus mass are lost by viscously driven outflows, neutrino-powered winds contribute at most another $\sim 1 \%$. Since BH-torus ejecta possess a wide distribution of electron fractions and entropies, they produce heavy elements from A 80 up to the actinides, with relative contributions of $A>130$ nuclei being subdominant. The combined ejecta of $\mathrm{CBM}$ and $\mathrm{BH}$-torus phases can reproduce the solar abundances amazingly well for $A>90$. Varying contributions of the torus ejecta might account for observed variations of lighter elements with $40<Z<56$ relative to heavier ones, and a considerable reduction of the prompt ejecta compared to the torus ejecta, e.g. in highly asymmetric NS-BH mergers, might explain the composition of heavy-element deficient stars.
\end{abstract}

XIII Nuclei in the Cosmos

7-11 July, 2014

Debrecen, Hungary

* Speaker. 


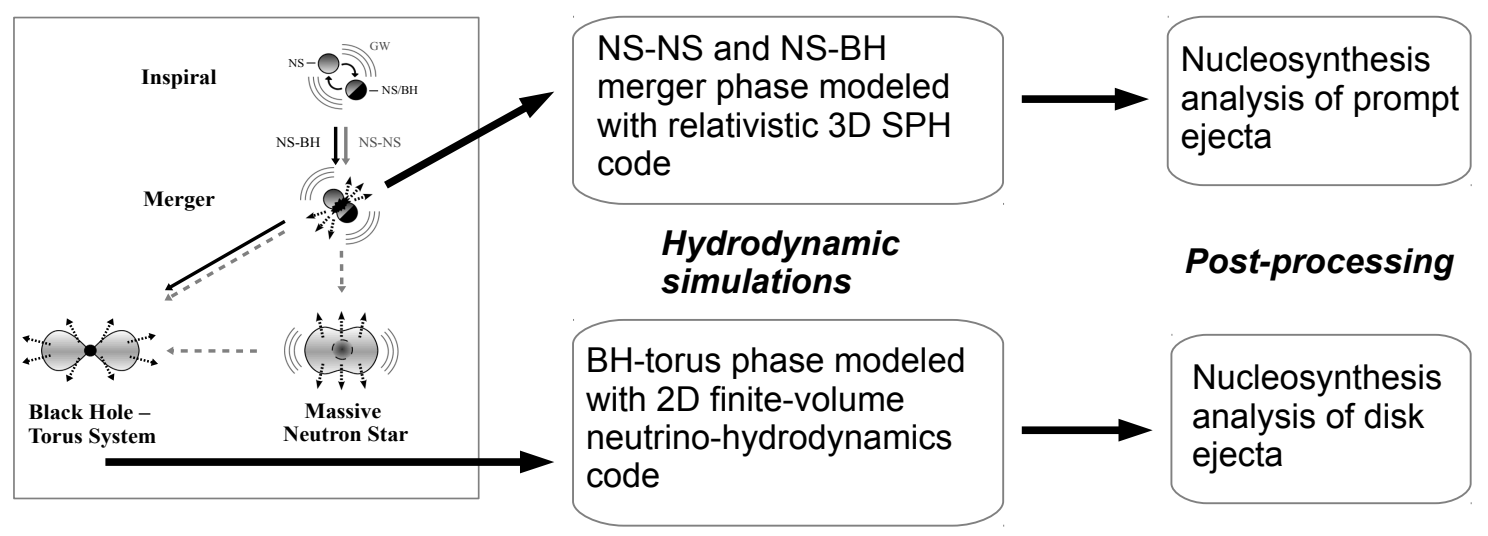

Figure 1: Schematical overview of our study. See main text for details.

\section{Motivation and Overview}

The astrophysical site(s) for the production of r-process elements is (are) still mysterious. Recent studies suggest that instead of or in addition to the long favored core-collapse supernovae the outflows from compact binary (i.e. double neutron-star, NS-NS, or neutron-star black-hole, NS-BH) mergers could provide suitable conditions to allow for the r-process. Most existing nucleosynthesis studies [1-6] only consider the prompt (dynamical) ejecta launched during the merger phase in a NS-NS configuration. However, also the dynamical ejecta from NS-BH mergers as well as the ejecta stemming from the remnants of both merger types need to be taken into account in order to understand the full chemogalactic fingerprint of compact object mergers. In both merger types, the merger remnant can consist of a BH surrounded by a more or less massive torus of neutron-star debris. In [7] we conducted a comprehensive analysis of the nucleosynthetic output associated with the ejecta from the merging phase of NS-NS and NS-BH binaries and with the outflows of the subsequent long-time evolution of relic BH-torus systems. We summarize the individual steps of this study in Fig. 1. The compact binary mergers are simulated with a relativistic smooth-particle-hydrodynamics code [8], while the BH-torus modeling is performed with a Eulerian finite-volume Godunov-type scheme [9], supplemented by a shear-viscosity treatment with a Shakura-Sunyaev $\alpha$-prescription for the dynamic viscosity [10]. For the long-time evolution we employ a pseudo-Newtonian approximation of the gravity potential for the rotating relic BHs [11]. In the time-dependent BH-torus modeling we apply, for the first time, detailed energy-dependent and velocity-dependent 2D neutrino transport based on a new two-moment closure scheme [12], which allows us to determine the neutrino-driven wind and the neutron-to-proton ratio in the disk outflows with higher accuracy than in previous simulations. All simulations include microphysical treatments of the gas equation of state (EOS).

Our nucleosynthesis calculations are carried out in a post-processing step of the ejecta produced by the hydrodynamical models, using a full r-process network including all relevant nuclear reactions [13-15]. For the combined analysis we pick cases from larger sets of NS-NS merger models, which lead to prompt or slightly delayed $\mathrm{BH}$ formation, and NS-BH merger models on the one side and $\mathrm{BH}$-torus models on the other side such that the macroscopic system parameters (BH and torus masses and BH spins of the merger remnants) match roughly on both sides. 

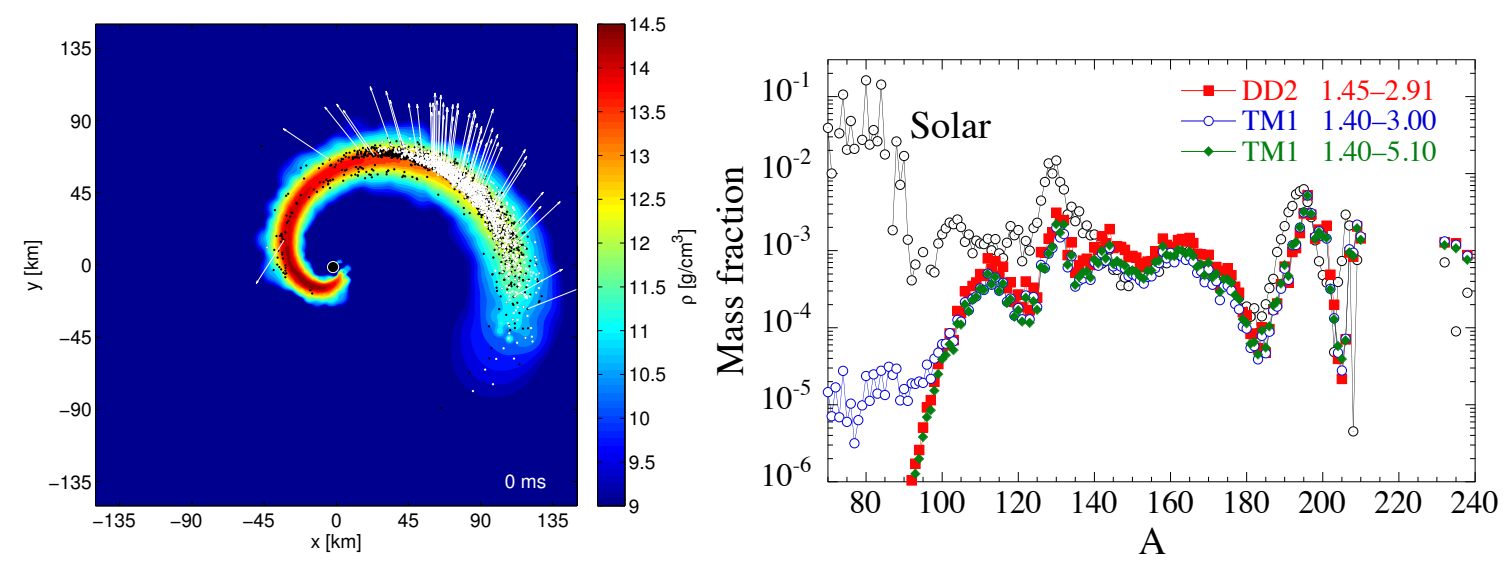

Figure 2: Left: Rest-mass density in the equatorial plane during the merger of a $1.4 M_{\odot} \mathrm{NS}$ with a $5.1 M_{\odot}$ $\mathrm{BH}$. Dots denote particles (projected into the equatorial plane) which are or will become gravitationally unbound while arrows indicate the corresponding velocities. The visualization tool SPLASH was used to convert SPH data to grid data [16]. Right: Abundance distributions as functions of the atomic mass for the dynamical ejecta of three NS-BH merger cases. Each binary system is characterized in the legend by the EOS used in the simulation and the mass (in $M_{\odot}$ ) of the NS and $\mathrm{BH}$, respectively. All distributions are normalized to the same A = 196 abundance. The dotted circles show the solar $r$-abundance distribution [17].

\section{Ejecta from the Merger Phase}

Consistent with previously published relativistic results $[5,18,6]$, the "dynamical" ejecta of NS-NS and NS-BH mergers, which are expelled within milliseconds of the collision of the two binary components, were found to possess similar average properties, namely expansion velocities of 0.2-0.4c, electron fractions below $\sim 0.1$, and entropies per baryon of a few $k_{\mathrm{B}}$. The considered NS-NS mergers produce $\sim 0.004-0.021 M_{\odot}$ of ejecta, whereas the NS-BH mergers eject significantly larger masses, $0.035-0.08 M_{\odot}$, with very low entropies ( $\lesssim 1 k_{\mathrm{B}}$ per nucleon), because this matter is not shock heated as in NS-NS collisions, but originates mostly from the outer tail of the tidally stretched NS at its final approach to the BH. Mass lost in NS-BH mergers is also expelled much more asymmetrically than in the case of NS-NS mergers (see left plot in Fig. 2): Corresponding hemispheric asymmetry parameters (mass difference between dominant ejecta hemisphere and opposite hemisphere, divided by total ejecta mass) are a few per cent for symmetric NS-NS mergers and $15-30 \%$ for strongly asymmetric ones, but $0.93-0.98$ for NS-BH mergers.

Since the high neutron excess, thermodynamic properties, and expansion timescale are very similar, the ejecta of NS-NS mergers as well as those of NS-BH mergers are sites of robust production of r-nuclei with $A \gtrsim 140$ and abundances close to the solar distribution (see right plot in Fig. 2 for typical abundance pattern resulting in the NS-BH case). This result holds basically independently of the considered nuclear EOS and the exact binary parameters and confirms the findings of previous studies based on relativistic merger simulations $[5,19,6]$.

\section{Ejecta from the BH-Torus Phase}

The relic BH-torus systems lose mass in neutrino-driven baryonic winds [20] and in outflows triggered by viscous energy dissipation and angular momentum transport [21-23]. We find that the 

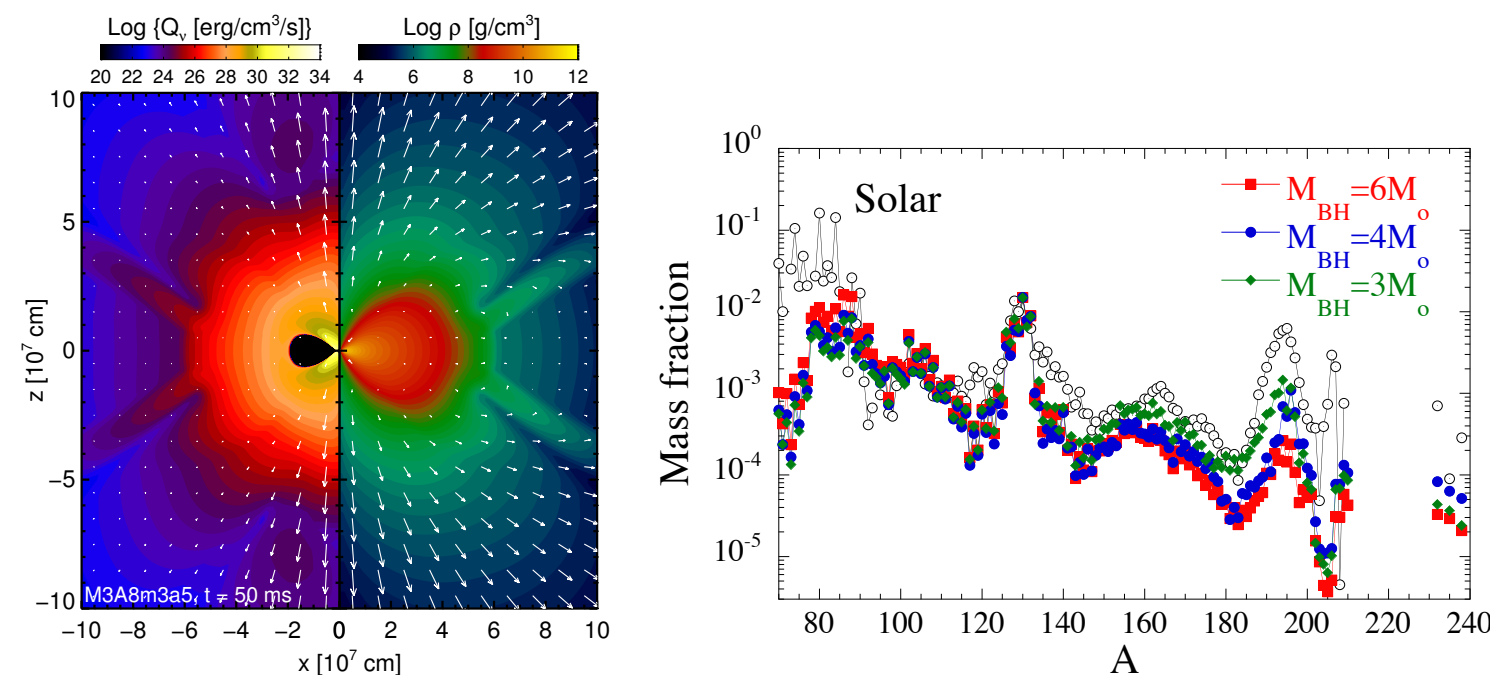

Figure 3: Left: Snapshot of a BH-torus model with a $3 M_{\odot}$ central $\mathrm{BH}$ with spin parameter 0.8 and an initial torus mass of $0.3 M_{\odot}$ at about $50 \mathrm{~ms}$ after the merger. On the left side the net neutrino heating rate due to $\beta$-processes and neutrino pair annihilation is color coded and overlaid with arrows indicating the energy-integrated flux density of electron neutrinos multiplied with the squared radius. On the right side the density is color coded and overlaid with velocity arrows. Right: Abundance distributions as functions of the atomic mass for three $\mathrm{BH}$-torus systems with $\mathrm{BH}$ masses of $M_{\mathrm{BH}}=3,4$ and $6 M_{\odot}$ and the same $0.3 M_{\odot}$ tori. All distributions are normalized to the same solar $A=130$ abundance. The dotted circles show the solar r-abundance distribution [17].

viscously driven ejecta amount up to, fairly model independent, $19-26 \%$ of the initial torus mass (for our $\mathrm{BH}$ spins of $A_{\mathrm{BH}}=0.8$ ) and dominate the neutrino-driven ejecta by far. The neutrino-driven wind depends extremely sensitively on the $\mathrm{BH}$ mass and on the torus mass, which determines the neutrino luminosities. The neutrino energy loss rates can reach several $10^{52} \mathrm{erg} \mathrm{s}^{-1}$ up to more than $10^{53} \mathrm{erg} \mathrm{s}^{-1}$ for each of $v_{e}$ and $\bar{v}_{e}$ for a few $100 \mathrm{~ms}$. The neutrino-driven ejecta carry away up to about one per cent of the initial torus mass, but their mass can also be orders of magnitude lower. The maximum masses that can be associated with ejecta driven by neutrino heating are 2.5-3.5 $\times 10^{-3} M_{\odot}$ in the case of high neutrino luminosities for typical durations of fractions of a second up to $\sim 1 \mathrm{~s}$. This number agrees with the lower limit of the expelled mass computed by [24] for a hypermassive NS as merger remnant, and the corresponding mass-loss rate is roughly compatible with the values obtained for neutrino winds of proto-neutron stars in supernova cores [25]. Neutrino heating, however, inflates the outer layers of the torus and has a positive feedback on the viscously driven mass ejection on the level of several per cent of the torus mass (or up to $\sim 20 \%$ of the viscous-outflow mass).

Neutrino-driven torus winds exhibit characteristic properties which distinguish them from the viscously triggered outflow, namely the tendency of higher mean entropies, higher electron fractions, and larger expansion velocities. Moreover, the neutrino wind is strongest at early times and at intermediate latitudes (around $45^{\circ}$ away from the equatorial plane), and the entropy, electron fraction, and velocity exhibit a strong pole-to-equator variation with higher values towards the poles. In contrast, viscously driven ejecta develop on longer timescales, are more spherical, and their properties vary little with angular direction. 

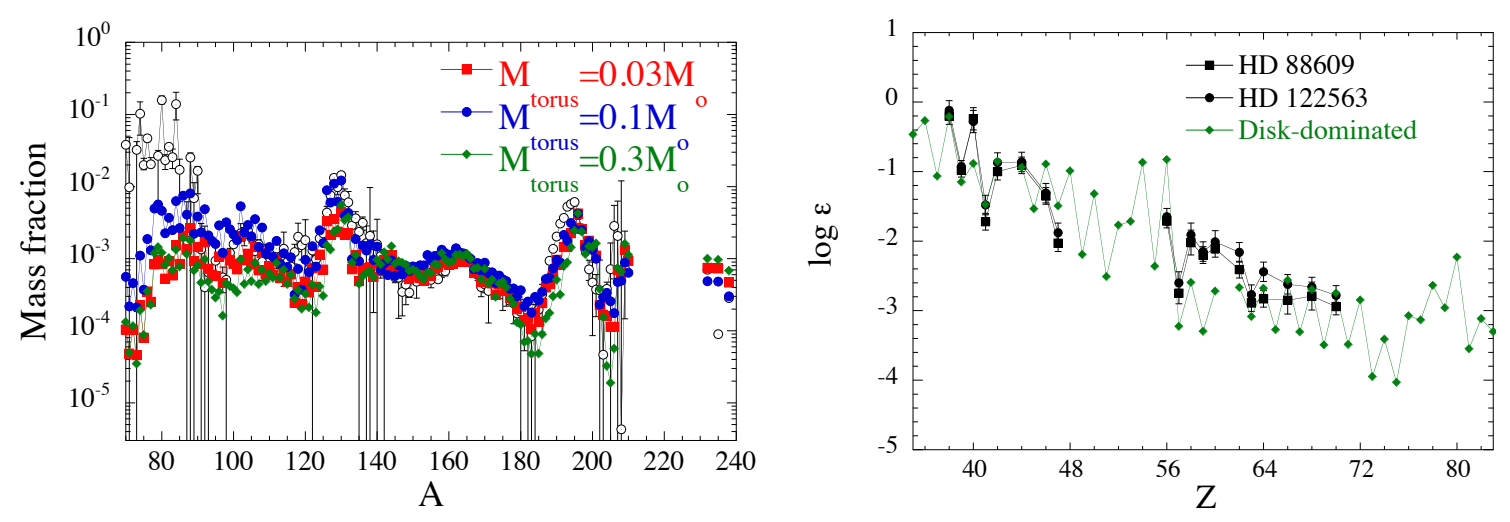

Figure 4: Left: Abundance distributions as functions of the atomic mass for three combined systems (merger model plus remnant model) corresponding to models with the indicated torus masses. All distributions are normalized to the same solar $A=196$ abundance. The dotted circles show the solar $\mathrm{r}$-abundance distribution [17]. Right: Comparison between the HD88609 and HD122563 elemental abundances (in $\log \varepsilon$ scale) and those estimated for the combination of a merger model and a BH-torus model, when the ejected mass of the merger model is assumed to be 100 times smaller than the one coming from the BH-torus model. The calculated distribution is normalized to the Sr abundance of HD88609.

Because of their greater neutron excess, viscously-driven ejecta allow for a much stronger r-process than the neutrino wind. The combination of both components is far dominated by the viscous contribution and matches the solar abundance pattern for all nuclear mass numbers $A \gtrsim 90$ fairly well. The abundance pattern for $A \leq 132$ is comparatively uniform, but the strength of the third abundance peak decreases with higher BH mass (see Fig. 3), and the relative yields of lowmass $(A \lesssim 130)$ and high-mass $(A \gtrsim 130)$ components depend on the value of the dynamic viscosity and the detailed treatment of the viscosity terms in the hydrodynamics equations.

\section{Combined Ejecta and Observational Implications}

The consistently mass-weighted combination of the prompt ejecta from the merger models and the secular ejecta from the BH-torus models can reproduce amazingly well the solar r-abundance pattern in the range $90 \lesssim A<240$ and therefore also that seen in ultra-metal-poor stars (cf. left plot in Fig. 4). In particular, the BH-torus outflows are able to well fill the region $A \lesssim 140$, where the prompt merger ejecta underproduce the nuclei (but see [6]). Since the relative yields of the relic BH-torus systems for $A \lesssim 130$ and $A \gtrsim 130$ nuclei depend sensitively on the system parameters, whereas the $A \gtrsim 140$ species are created with a robust pattern during the binary merging phase, we expect a larger variability in the low- $A$ regime than for high mass numbers.

Another interesting possibility is connected to the fact that the mass ejection during NS-BH mergers shows extreme spatial asymmetry (corresponding to asymmetry parameters $\gtrsim 0.95$ ) but the mass loss from their BH-torus remnants is much more isotropic. This can lead to a strong suppression of the dynamical ejecta component relative to the torus outflow in observer directions pointing away from the hemisphere that receives most of the expelled matter of the disrupted NS. Essentially pure BH-torus ejecta of some of our models can reasonably well match the abundance 
distributions observed in heavy-element deficient metal-poor stars like HD88609 and HD122563 (right plot in Fig 4).

Since the major part of the torus ejecta ends up in forming $A \lesssim 130$ material, the additional mass loss of the merger remnants does not alter event-rate estimates based on comparing yields of heavy r-nuclei in the ejecta of the binary-merger phase with the r-process abundances in our Galaxy $[2,26]$.

\section{Acknowledgements}

At Garching, this research was supported by the Max-Planck/Princeton Center for Plasma Physics (MPPC) and by the Deutsche Forschungsgemeinschaft through the SFB-TR7 and the Cluster of Excellence EXC 153. AB is a Marie Curie Intra-European Fellow within the 7th European Community Framework Programme (IEF 331873). SG acknowledges financial support from FNRS (Belgium). We are also grateful for computational support by the Center for Computational Astrophysics (C2PAP) at the Leibniz Rechenzentrum (LRZ) and by the Rechenzentrum Garching (RZG).

\section{References}

[1] C. Freiburghaus, S. Rosswog, and F.-K. Thielemann, R-Process in Neutron Star Mergers, ApJL 525 (Nov., 1999) L121-L124.

[2] S. Goriely, A. Bauswein, and H.-T. Janka, r-process Nucleosynthesis in Dynamically Ejected Matter of Neutron Star Mergers, ApJL 738 (Sept., 2011) L32+, [arXiv: 1107.0899$].$

[3] L. F. Roberts, D. Kasen, W. H. Lee, and E. Ramirez-Ruiz, Electromagnetic Transients Powered by Nuclear Decay in the Tidal Tails of Coalescing Compact Binaries, ApJL 736 (July, 2011) L21+, [arXiv:1104.5504].

[4] O. Korobkin, S. Rosswog, A. Arcones, and C. Winteler, On the astrophysical robustness of the neutron star merger r-process, MNRAS 426 (Nov., 2012) 1940-1949, [arXiv: 1206.2379 ].

[5] A. Bauswein, S. Goriely, and H.-T. Janka, Systematics of Dynamical Mass Ejection, Nucleosynthesis, and Radioactively Powered Electromagnetic Signals from Neutron-star Mergers, ApJ 773 (Aug., 2013) 78, [arXiv:1302.6530].

[6] S. Wanajo, Y. Sekiguchi, N. Nishimura, K. Kiuchi, K. Kyutoku, and M. Shibata, Production of All the r-process Nuclides in the Dynamical Ejecta of Neutron Star Mergers, ApJL 789 (July, 2014) L39, [arXiv:1402.7317].

[7] O. Just, A. Bauswein, R. Ardevol Pulpillo, S. Goriely, and H.-T. Janka, Comprehensive nucleosynthesis analysis for ejecta of compact binary mergers, ArXiv e-prints (June, 2014) [arXiv:1406.2687].

[8] R. Oechslin, H. Janka, and A. Marek, Relativistic neutron star merger simulations with non-zero temperature equations of state. I. Variation of binary parameters and equation of state, A\&A $\mathbf{4 6 7}$ (May, 2007) 395-409, [astro-ph/ 0611047 ].

[9] M. Obergaulinger, Astrophysical magnetohydrodynamics and radiative transfer: numerical methods and applications. $\mathrm{PhD}$ thesis, Technical University Munich, 2008. 
[10] N. I. Shakura and R. A. Sunyaev, Black holes in binary systems. Observational appearance., A\&A 24 (1973) 337-355.

[11] I. V. Artemova, G. Bjoernsson, and I. D. Novikov, Modified Newtonian Potentials for the Description of Relativistic Effects in Accretion Disks around Black Holes, ApJ 461 (Apr., 1996) 565-+.

[12] O. Just, M. Obergaulinger, and H. T. Janka, A new multidimensional, energy-dependent, two-moment transport code for neutrino-hydrodynamics, in preparation.

[13] S. Goriely, S. Hilaire, and A. J. Koning, Improved predictions of nuclear reaction rates with the TALYS reaction code for astrophysical applications, A\&A 487 (Aug., 2008) 767-774, [arXiv:0806.2239].

[14] S. Goriely, N. Chamel, and J. M. Pearson, Further explorations of Skyrme-Hartree-Fock-Bogoliubov mass formulas. XII. Stiffness and stability of neutron-star matter, Phys. Rev. C 82 (Sept., 2010) 035804.

[15] Y. Xu, S. Goriely, A. Jorissen, G. L. Chen, and M. Arnould, Databases and tools for nuclear astrophysics applications. BRUSsels Nuclear LIBrary (BRUSLIB), Nuclear Astrophysics Compilation of REactions II (NACRE II) and Nuclear NETwork GENerator (NETGEN), A\&A 549 (Jan., 2013) A106.

[16] D. J. Price, splash: An Interactive Visualisation Tool for Smoothed Particle Hydrodynamics Simulations, PASA 24 (Oct., 2007) 159-173, [arXiv:0709.0832].

[17] S. Goriely, Uncertainties in the solar system r-abundance distribution, A\&A 342 (Feb., 1999) 881-891.

[18] F. Foucart, M. B. Deaton, M. D. Duez, E. O'Connor, C. D. Ott, R. Haas, L. E. Kidder, H. P. Pfeiffer, M. A. Scheel, and B. Szilagyi, Neutron star-black hole mergers with a nuclear equation of state and neutrino cooling: Dependence in the binary parameters, Phys. Rev. D 90 (July, 2014) 024026, [arXiv:1405.1121].

[19] K. Hotokezaka, K. Kiuchi, K. Kyutoku, H. Okawa, Y.-i. Sekiguchi, M. Shibata, and K. Taniguchi, Mass ejection from the merger of binary neutron stars, Phys. Rev. D 87 (Jan., 2013) 024001, [arXiv:1212.0905].

[20] S. Wanajo and H.-T. Janka, The r-process in the Neutrino-driven Wind from a Black-hole Torus, ApJ 746 (Feb., 2012) 180, [arXiv:1106.6142].

[21] R. Fernández and B. D. Metzger, Delayed outflows from black hole accretion tori following neutron star binary coalescence, MNRAS 435 (Oct., 2013) 502-517, [arXiv: 1304 . 6720].

[22] B. D. Metzger and R. Fernández, Red or blue? A potential kilonova imprint of the delay until black hole formation following a neutron star merger, MNRAS 441 (July, 2014) 3444-3453, [arXiv:1402.4803].

[23] R. Fernández, D. Kasen, B. D. Metzger, and E. Quataert, Outflows from accretion disks formed in neutron star mergers: effect of black hole spin, ArXiv e-prints (Sept., 2014) [arXiv: 1409.4426 ].

[24] A. Perego, S. Rosswog, R. M. Cabezón, O. Korobkin, R. Käppeli, A. Arcones, and M. Liebendörfer, Neutrino-driven winds from neutron star merger remnants, MNRAS 443 (Oct., 2014) 3134-3156, [arXiv:1405.6730].

[25] Y. Qian and S. E. Woosley, Nucleosynthesis in Neutrino-driven Winds. I. The Physical Conditions, ApJ 471 (Nov., 1996) 331-+, [astro-ph/9611094].

[26] A. Bauswein, R. Ardevol Pulpillo, H.-T. Janka, and S. Goriely, Nucleosynthesis Constraints on the Neutron Star-Black Hole Merger Rate, ApJL 795 (Nov., 2014) L9, [arXiv : 1408 . 1783]. 